\title{
Potato Antioxidant Compounds: Impact of Cultivation Methods and Relevance for Diet and Health
}

\author{
Danièle Evers and Hannah Deußer \\ Environment and Agro-Biotechnologies Department, \\ Centre de Recherche Public - Gabriel Lippmann, Belvaux \\ Luxembourg
}

\section{Introduction}

Potato is currently the fourth most important food crop in the world after maize, wheat and rice, with a production of 329 millions tons (FAO, 2009). As for the harvested area, potato ranks $7^{\text {th }}$ after wheat, rice, maize, barley, sorghum and rapeseed worldwide. In terms of consumption, potato ranks third after rice and wheat. Interestingly, the importance of potato in the diet is higher in developed as compared to developing countries, accounting for $130 \mathrm{kcal}$ per person per day for the developed world and for $41 \mathrm{kcal}$ per person per day in the developing world (Burlingame et al., 2009). In Europe, the per capita consumption reaches almost $90 \mathrm{~kg}_{\text {year-1, }}$, whereas in developing countries, its per capita consumption is smaller, reaching around $20 \mathrm{~kg}_{\text {year-1 }}$ (André et al., 2007a, FAO, 2009)).

Potato is mainly known to supply dietary fibre, carbohydrates, high-quality proteins, vitamins and minerals. According to the USDA National Nutrient Database, contents would be $2.4 \mathrm{~g}$ for dietary fiber, $15.7 \mathrm{~g}$ for carbohydrates, $1.7 \mathrm{~g}$ for protein content, $19.7 \mathrm{~g}$ for vitamin C, each per $100 \mathrm{~g}$ of white, raw potato (flesh and skin) (Singh \& Kaur, 2009). As for the minerals, iron and zinc contents are around $0.52 \mathrm{mg}$ and $0.29 \mathrm{mg}$ per $100 \mathrm{~g}$ (Singh \& Kaur, 2009). Potatoes are also known as sources of antioxidant compounds, including polyphenols, carotenoids and vitamins, pointing to their relevance not only as a starchy food, but also as a vegetable.

Data on the antioxidant content of potatoes can be found in several national food composition tables. Table 1 summarizes some of the data available on contents of ascorbic acid, $\beta$-carotene, a-tocopherol, lutein and zeaxanthin. Phenolic compounds were not specified in these databases.

Potato's genetic diversity is huge; more than 4000 different wild varieties have been collected at the International Potato Center in Lima (Peru). They are diverse regarding tuber shape, flesh and skin colour, flavour, storage quality and cooking quality (André et al., 2007a).

The classification of cultivated potatoes has been reviewed several times and still is a matter of debate. Huaman and Spooner (2002) classified all landrace populations of cultivated 


\begin{tabular}{lcccccc}
\hline Compound & DK & F & D & CH & USA white & USA red \\
\hline Ascorbic acid $(\mathrm{mg} / 100 \mathrm{~g})$ & 26.4 & 11.1 & 18.8 & 17.0 & 19.7 & 8.6 \\
$\beta$-Carotene $(\mu \mathrm{g} / 100 \mathrm{~g})$ & 10 & 2 & 5 & 5 & 5 & 4 \\
Lutein + Zeaxanthin $(\mu \mathrm{g} / 100 \mathrm{~g})$ & n.a. & n.a. & n.a. & n.a. & 13 & 21 \\
a-Tocopherol $(\mathrm{mg} / 100 \mathrm{~g})$ & 0.10 & $0.05^{*}$ & 0.05 & $0.06^{*}$ & 0.01 & 0.01 \\
\hline
\end{tabular}

Table 1. Antioxidant contents of potatoes. Data were obtained from national food composition tables. DK = potato, raw, Denmark (National Food Institute - Technical University of Denmark, 2009), F = potato, boiled, France (French Information Center on Food Quality, 2008); D = potato, peeled, raw, Germany (Max-Rubner-Institut - Karlsruhe, 2010); $\mathrm{CH}=$ potato, peeled, raw, Switzerland (ETH Zürich, 2009); USA white = potato, white, raw, U.S.A. (Nutrient Data Laboratory, 2010); USA red = potato, red, raw, U.S.A. (Nutrient Data Laboratory, 2010); n.a. = not analyzed; * = a-tocopherol equivalents.

potatoes in a single species Solanum tuberosum with 8 cultivar groups: Ajanhuiri Group (2x), Andigenum Group (4x), Caucha Group (3x), Chilotanum Group (4x), Curtilobum Group (5x), Juzepczukii Group (3x), Phureja Group (2x) and Stenotonum Group (2x). Modern cultivars were classified into a ninth cultivar group, the Tuberosum Group. However, it is worth mentioning that Spooner et al. (2007) reviewed the classification combining morphological data with molecular fingerprinting data.

In South America, the center of potato origin and diversity, potato constitutes the main staple crop and farmers cultivate up to 50 varieties in a field (FAO, 2008). This not only enables them to be protected from a complete loss in the case of a disease or an abiotic stress, it also allows them to get a more diversified diet. Indeed, the composition of the tubers varies according to the cultivar, agricultural practices, climate and soil (Rodriguez et al., 2010); moreover cooking and processing may have an effect on tuber composition (Xu et al., 2009).

Biofortification programs aiming at enriching nutrient contents of edible plants for health improvement and disease prevention are ongoing. They include biofortification through fertilization, breeding or biotechnology (White \& Broadley, 2005). Nutrient biofortification of food crops may not only include elevated mineral and amino acid levels, but also enhanced antioxidant levels (Diretto et al., 2007, Rommens et al., 2008). Traditional agricultural approaches, such as breeding, can improve the nutritional value to some extent (Hirschi, 2009); especially if the corresponding trait is strongly dependent on the genotype, the selection of adequate progenitors allows to expect good progress in breeding (Burgos et al., 2009a).

The present chapter gives an overview on the potato antioxidants and on parameters impacting their contents in the tuber. Moreover, it will give an insight into potential healthpromoting effects and bioavailability of antioxidants.

\section{Antioxidants in potato}

The concept of potato as a source of antioxidants is not widely spread. However, recent studies have placed potato into the perspective of an antioxidant-rich crop. More precisely, potatoes contain phenolic compounds including hydroxycinnamic acids, the predominant being chlorogenic acid (André et al., 2007b, Brown, 2005) and flavonoids, for example 
catechin, epicatechin and anthocyanins. Potato contains low amounts of carotenoids, such as $\beta$-carotene (Brown, 2005), indicating that potato is not a good source of pro-vitamin A carotenes; more important are the oxygenated carotenoids, the xantophylls, such as neoxanthin, violaxanthin, antheraxanthin, lutein and zeaxanthin (Griffiths et al., 2007). As for the vitamins, potato contains on average $20 \mathrm{mg}$ per $100 \mathrm{~g}$ FW of vitamin C (Brown, 2005) and concerning vitamin $\mathrm{E}$, mainly $\alpha$-tocopherol is present at concentrations between 55 and $416 \mu \mathrm{g}$ per $100 \mathrm{~g}$ FW (André et al., 2007b). Hereafter, the types and contents of potato antioxidants will be discussed in more detail.

\subsection{Polyphenols}

Polyphenols are secondary plant metabolites. In potatoes, mainly hydroxycinnamic acid derivatives and flavonoids occur. As examples, the chemical structures of chlorogenic acid, a hydroxycinnamic acid ester with quinic acid, and kaempferol 3-O-rutinoside, a flavonol glycoside, are shown in Figure 1.

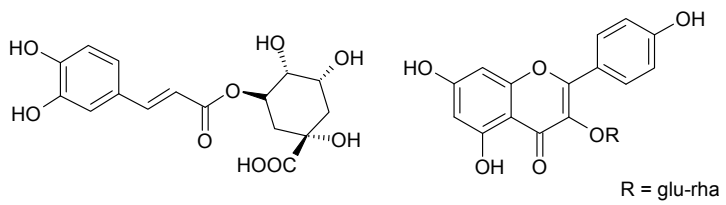

Fig. 1. Chemical structures of chlorogenic acid (left) and kaempferol 3-O-rutinoside (right).

\begin{tabular}{|c|c|c|c|}
\hline Plant material & $\begin{array}{c}\text { Total phenolic } \\
\text { content (mg/g DW) }\end{array}$ & Sample preparation & Reference \\
\hline S. tuberosum & $2.5-4.8$ & crude, freeze-dried & (Lachman et al., 2008) \\
\hline unspecified & $4.5-6.8$ & crude, freeze-dried & (Stratil et al., 2006) \\
\hline Solanum sp. & $1.8-11$ & crude, freeze-dried & (Navarre et al., 2011) \\
\hline unspecified & $0.9-3.0$ & crude, freeze-dried & (Xu et al., 2009) \\
\hline S. tuberosum & $1.1-27.4^{a}$ & crude, freeze-dried & $\begin{array}{l}\text { (André et al., 2007a, } \\
\text { André et al., 2009a) }\end{array}$ \\
\hline unspecified & $0.32^{\mathrm{b}}$ & crude & (Chiou et al., 2007) \\
\hline S. tuberosum & $0.3-0.5$ & crude, freeze-dried & (Rumbaoa et al., 2009) \\
\hline S. tuberosum & 15.1 & crude & (Natella et al., 2010) \\
\hline unspecified & $0.6-2.3^{a}$ & crude & (Blessington et al., 2010) \\
\hline Solanum sp. & $3-16 a$ & crude & (Campos et al., 2006) \\
\hline S. tuberosum & $1.5-3.2$ & cooked, peeled, mashed & (Kaspar et al., 2011) \\
\hline S. tuberosum & $2.1-7.8$ a & crude & (Reddivari et al., 2007) \\
\hline S. tuberosum & $1.0-2.9$ & crude, freeze-dried & (Leo et al., 2008) \\
\hline S. tuberosum & $1.3-13.4$ & crude & (Madiwale et al., 2011) \\
\hline S. tuberosum & 1.8 & crude, freeze-dried & (Mäder et al., 2009) \\
\hline S. tuberosum & $8-78$ & crude, freeze-dried & (Stushnoff et al., 2010) \\
\hline
\end{tabular}

Table 2. Total phenolic content of potatoes determined with the Folin-Ciocalteau assay. As far as not otherwise indicated, the results are expressed as gallic acid equivalents. a As chlorogenic acid equivalents, $\mathrm{b}$ as caffeic acid equivalents. Contents reported in another unit than $\mathrm{mg} / \mathrm{g}$ dry weight (DW) were recalculated utilizing an average moisture content of $80 \%$. 
Here, we present an overview on current available literature on total phenolic contents (Table 2), anthocyanins (Table 3) and individual polyphenols (Table 4). Total phenolic contents have been determined by using a spectrophotometric assay, the Folin-Ciocalteau assay. This assay estimates the phenolic content in plants, but other plant compounds (for example thiol derivatives, vitamins, and amino acids) may also be detected (Everette et al., 2010). Total anthocyanins, which impart for colour in red and purple potatoes, have been determined using the $\mathrm{pH}$ differential method (Giusti \& Wrolstad, 2001). This method gives the sum of all anthocyanins present in the sample. Individual polyphenols have been quantified by HPLC with UV-detection.

\begin{tabular}{|c|c|c|c|}
\hline Plant material & $\begin{array}{l}\text { Total anthocyanin content } \\
(\mathrm{mg} / \mathrm{g} \text { DW) }\end{array}$ & $\begin{array}{c}\text { Sample } \\
\text { preparation }\end{array}$ & Reference \\
\hline S. tuberosum & $0-21.4^{a}$ & crude, freeze-dried & $\begin{array}{l}\text { (André et al., 2009a, } \\
\text { André et al., 2009b) }\end{array}$ \\
\hline S. tuberosum & $0.3-1.8 b$ & crude, freeze-dried & (Brown, 2008) \\
\hline Solanum sp. & $0-4 b$ & crude & (Campos et al., 2006) \\
\hline S. tuberosum & $0-6.2$ & $\begin{array}{l}\text { cooked, peeled, } \\
\text { mashed }\end{array}$ & (Kaspar et al., 2011) \\
\hline Solanum sp. & $0-1.2 b$ & crude & (Brown et al., 2007) \\
\hline S. tuberosum & $1.0-5.5$ & crude & (Madiwale et al., 2011) \\
\hline S. tuberosum & $2-45$ & crude, freeze-dried & (Stushnoff et al., 2010) \\
\hline
\end{tabular}

Table 3. Total anthocyanin content of potatoes determined with the $\mathrm{pH}$ differential method. The results are expressed as apetanin or ${ }^{b}$ cyanidin 3-O-glucoside. In some references the standard compound was not stated. Contents reported in another unit than $\mathrm{mg} / \mathrm{g}$ dry weight (DW) were recalculated utilizing an average moisture content of $80 \%$.

\begin{tabular}{|c|c|c|c|}
\hline Plant material & $\begin{array}{l}\text { Phenolic content } \\
(\mu \mathrm{g} / \mathrm{g} \text { DW })\end{array}$ & Sample preparation & Reference \\
\hline \multicolumn{4}{|l|}{ Chlorogenic acid } \\
\hline $\begin{array}{l}\text { S. tuberosum crs. } \\
\text { Siikli and Timo }\end{array}$ & $455-600$ & cooked, freeze-dried & $\begin{array}{l}\text { (Mattila \& Hellström, } \\
\text { 2007) }\end{array}$ \\
\hline $\begin{array}{l}\text { S. tuberosum cv. } \\
\text { Désirée }\end{array}$ & 1500 & crude, freeze-dried & $\begin{array}{l}\text { (Lukaszewicz et al., } \\
\text { 2004) }\end{array}$ \\
\hline S. tuberosum cv. Sava & $361-520$ & $\begin{array}{l}\text { crude, peeled, } \\
\text { freeze-dried }\end{array}$ & (Soltoft et al., 2010) \\
\hline S. tuberosum & $421-2185$ & crude, freeze-dried & (Zhu et al., 2010) \\
\hline Solanum sp. & $220-4730$ & crude, freeze-dried & (Navarre et al., 2011) \\
\hline Solanum sp. & $55-1340$ & crude & $\begin{array}{l}\text { (del Mar Verde Mendez } \\
\text { et al., 2004) }\end{array}$ \\
\hline unspecified & $420.5-3183.4$ & crude, freeze-dried & (Xu et al., 2009) \\
\hline S. tuberosum & $216-12746$ & crude, freeze-dried & $\begin{array}{l}\text { (André et al., 2007b, } \\
\text { André et al., 2009a, } \\
\text { André et al., 2009b) }\end{array}$ \\
\hline unspecified & 6.5 & crude & (Chiou et al., 2007) \\
\hline unspecified & $33-6370$ & crude, freeze-dried & (Im et al., 2008) \\
\hline S. tuberosum & $600-2100$ & crude, freeze-dried & (Navarre et al., 2010) \\
\hline
\end{tabular}




\begin{tabular}{|c|c|c|c|}
\hline Plant material & $\begin{array}{l}\text { Phenolic content } \\
(\mu \mathrm{g} / \mathrm{g} \text { DW })\end{array}$ & Sample preparation & Reference \\
\hline $\begin{array}{l}\text { S. tuberosum cr. } \\
\text { Norkotah and Ranger }\end{array}$ & 2200 and 1000 & crude, freeze-dried & $\begin{array}{l}\text { (Shakya \& Navarre, } \\
\text { 2006) }\end{array}$ \\
\hline S. tuberosum & $343-1249$ & crude & (Hajslova et al., 2005) \\
\hline unspecified & $39.5-65$ & crude & (Blessington et al., 2010) \\
\hline S. tuberosum & $470-920$ & crude, freeze-dried & (Leo et al., 2008) \\
\hline S. tuberosum & 718 & crude, freeze-dried & (Mäder et al., 2009) \\
\hline \multicolumn{4}{|l|}{ Caffeic acid } \\
\hline $\begin{array}{l}\text { S. tuberosum cv. } \\
\text { Désirée }\end{array}$ & 800 & crude, freeze-dried & $\begin{array}{l}\text { (Lukaszewicz et al., } \\
\text { 2004) }\end{array}$ \\
\hline Solanum & $5-476$ & crude, freeze-dried & (Navarre et al., 2011) \\
\hline Solanum sp. & $5-135$ & crude & $\begin{array}{l}\text { (del Mar Verde Mendez } \\
\text { et al., 2004) }\end{array}$ \\
\hline unspecified & $0-93.8$ & crude, freeze-dried & (Xu et al., 2009) \\
\hline S. tuberosum & $7-143$ & crude, freeze-dried & $\begin{array}{l}\text { (André et al., 2007b, } \\
\text { André et al., 2009a, } \\
\text { André et al., 2009b) }\end{array}$ \\
\hline unspecified & $5-293$ & crude, freeze-dried & (Im et al., 2008) \\
\hline $\begin{array}{l}\text { S. tuberosum cV. } \\
\text { Norkotah and Ranger }\end{array}$ & 100 and 200 & crude, freeze-dried & $\begin{array}{l}\text { (Shakya \& Navarre, } \\
\text { 2006) }\end{array}$ \\
\hline S. tuberosum & $50-120$ & crude, freeze-dried & (Leo et al., 2008) \\
\hline S. tuberosum & 203 & crude, freeze-dried & (Mäder et al., 2009) \\
\hline \multicolumn{4}{|l|}{$\begin{array}{l}\text { Quercetin 3-O-rutinoside } \\
\text { (= Rutin) }\end{array}$} \\
\hline $\begin{array}{l}\text { S. tuberosum cv. } \\
\text { Monalisa }\end{array}$ & 180 & crude & (Tudela et al., 2002) \\
\hline Solanum sp. & $0-141$ & crude, freeze-dried & (Navarre et al., 2011) \\
\hline S. tuberosum & $0-256$ & crude, freeze-dried & $\begin{array}{l}\text { (André et al., 2007b, } \\
\text { André et al., 2009a, } \\
\text { André et al., 2009b) }\end{array}$ \\
\hline S. tuberosum & $6.6-7.4$ & crude, freeze-dried & (Navarre et al., 2010) \\
\hline unspecified & $26.5-49$ & crude & (Blessington et al., 2010) \\
\hline \multicolumn{4}{|l|}{$\begin{array}{l}\text { Kaempferol 3-O- } \\
\text { rutinoside }\end{array}$} \\
\hline Solanum sp. & $0-433$ & crude, freeze-dried & (Navarre et al., 2011) \\
\hline S. tuberosum & $0-227$ & crude, freeze-dried & $\begin{array}{l}\text { (André et al., 2007b, } \\
\text { André et al., 2009a, } \\
\text { André et al., 2009b) }\end{array}$ \\
\hline $\begin{array}{l}\text { S. tuberosum } \\
(+) \text {-Catechin } \\
\end{array}$ & $5.5-7.0$ & crude, freeze-dried & (Navarre et al., 2010) \\
\hline Solanum sp. & $90-1305$ & crude & $\begin{array}{l}\text { (del Mar Verde Mendez } \\
\text { et al., 2004) }\end{array}$ \\
\hline S. tuberosum & $430-1570$ & crude, freeze-dried & (Leo et al., 2008) \\
\hline S. tuberosum & 13 & crude, freeze-dried & (Mäder et al., 2009) \\
\hline
\end{tabular}

Table 4. Content of individual polyphenols in potatoes determined by HPLC-UV. Contents reported in another unit than $\mu \mathrm{g} / \mathrm{g}$ dry weight (DW) were recalculated utilizing an average moisture content of $80 \%$. 
As can be seen in tables 2, 3 and 4, potatoes contain between 0.3 and $78 \mathrm{mg} / \mathrm{g}$ DW of phenolic compounds, whereas the total anthocyanin content was shown to be 0 in white or yellow fleshed potatoes and up to $45 \mathrm{mg} / \mathrm{g}$ DW in some red and purple fleshed cultivars. Regarding the individual polyphenols, chlorogenic acid is the predominant compound in potatoes with contents ranging from 6.5 to $12746 \mu \mathrm{g} / \mathrm{g}$ DW. The contents of caffeic acid, quercetin 3-O-rutinoside, kaempferol 3-O-rutinoside and (+)-catechin ranged from 0 to 800 $\mu \mathrm{g} / \mathrm{g}$ DW, 0 to $256 \mu \mathrm{g} / \mathrm{g}$ DW, 0 to $433 \mu \mathrm{g} / \mathrm{g}$ DW, and 13 to $1570 \mu \mathrm{g} / \mathrm{g}$ DW, respectively. Generally, purple and red fleshed cultivars contained higher amounts of polyphenols than cultivars with a cream or white flesh.

Additionally to the phenolic compounds listed in Table 4, other compounds have been described, namely neochlorogenic acid, cryptochlorogenic acid, caffeoyl putrescine, $p$ coumaric acid, ferulic acid, cinnamic acid, syringic acid, sinapic acid, gallic acid, vanillin, vanillic acid, $p$-hydroxybenzoic acid, $p$-hydroxyphenylacetic acid, myricetin, (-)-epicatechin, p-coumaroylhydroxyagmatine, and petanin (André et al., 2007b, André et al., 2009b, Blessington et al., 2010, Chiou et al., 2007, del Mar Verde Mendez et al., 2004, Mäder et al., 2009, Navarre et al., 2011).

\subsection{Carotenoids}

The carotenoid levels in potatoes determine whether the tuber flesh is white (low carotenoid content), yellow (moderate content), or orange (high content). Carotenoids in potatoes belong to the groups of the bicyclic carotenes and the xanthophylls. Carotenes (e.g. $\beta$ carotene) are pure polyen hydrocarbons whereas xanthophylls (e.g. lutein) contain oxygen groups (hydroxyl-, epoxy-, or carbonyl-groups). The chemical structures of two carotenoids from potatoes are shown in Figure 2.

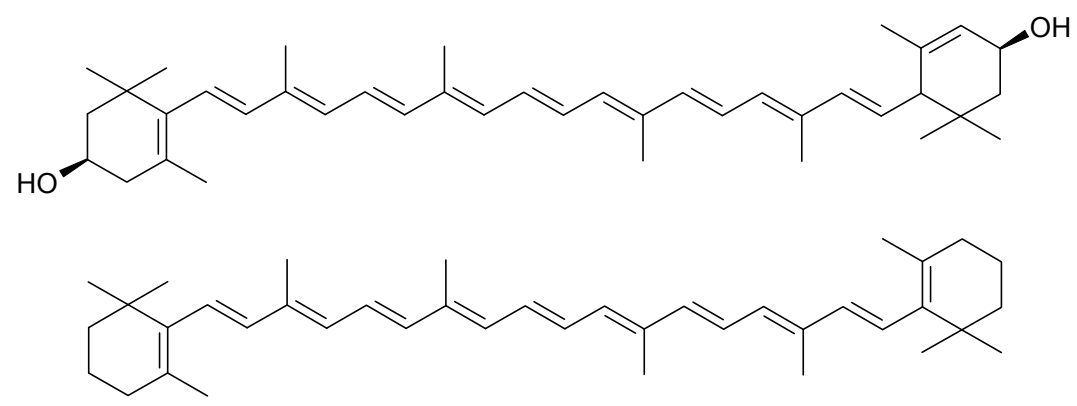

Fig. 2. Chemical structures of the carotenoids lutein (above) and $\beta$-carotene (below).

The total carotenoid contents and concentrations of individual carotenoids reported in literature are summarized in Table 5 and Table 6. To achieve a better comparability, values that were not expressed in $\mu \mathrm{g} / \mathrm{g}$ DW were recalculated to this basis. The major carotenoids in potatoes are violaxanthin, lutein, zeaxanthin, neoxanthin, and antheraxanthin, but according to the publications cited in Table 6 their contribution varies among the cultivars. The composition of carotenoids in the skin and the flesh of the potato tubers is similar (Burmeister et al., 2011). 
The determination of the total carotenoid content was mostly conducted in saponificated carotenoid extracts using a spectrophotometric assay, whereas individual carotenoids were identified and quantified by HPLC-UV analysis. Near-infrared reflectance spectroscopy (NIRS) - an analytical method that is less expensive and more rapid than HPLC - was also applied for the determination of individual carotenoids (Bonierbale et al., 2009).

\begin{tabular}{|c|c|c|c|}
\hline Plant material & $\begin{array}{c}\text { Carotenoid } \\
\text { content }(\mu \mathrm{g} / \mathrm{g} \text { DW) }\end{array}$ & Sample preparation & Reference \\
\hline S. tuberosum & $2.5-17.5$ & crude & (Brown, 2008) \\
\hline S. tuberosum & $3.9-7$ & crude & (Blessington et al., 2010) \\
\hline S. tuberosum & $10-25$ & crude & (Campos et al., 2006) \\
\hline $\begin{array}{l}\text { S. tuberosum } \\
\text { cv. Pentland } \\
\text { Javelin }\end{array}$ & 1.6 & $\begin{array}{l}\text { crude, freeze-dried, white } \\
\text { fleshed }\end{array}$ & (Morris et al., 2004) \\
\hline $\begin{array}{l}\text { S. tuberosum } \\
\text { cv. Desiree }\end{array}$ & 4.9 & $\begin{array}{l}\text { crude, freeze-dried, } \\
\text { cream/yellow-fleshed }\end{array}$ & (Morris et al., 2004) \\
\hline $\begin{array}{l}\text { S. phureja cv. } \\
\text { DB375 } \backslash 1\end{array}$ & 36.3 & crude, freeze-dried & (Morris et al., 2004) \\
\hline S. tuberosum & $1.9-43.9$ & crude & (Nesterenko \& Sink, 2003) \\
\hline $\begin{array}{l}\text { S. tuberosum } \\
\text { cv. Yukon Gold } \\
\text { and Superior }\end{array}$ & 3.2 and 5.6 & crude, without skin & (Lu \& Haynes, 2001) \\
\hline $\begin{array}{l}\text { Hybrid from S. } \\
\text { phureja and } S \text {. } \\
\text { stenotomum }\end{array}$ & 6.8 and 71.8 & crude, without skin & (Lu \& Haynes, 2001) \\
\hline S. tuberosum & $7.5-233$ & crude & (Kotikova et al., 2007) \\
\hline $\begin{array}{l}\text { Cross between } \\
\text { S. tuberosum } \\
\text { and S. phureja }\end{array}$ & $17.7-34.9$ & & (Kobayashi et al., 2008) \\
\hline S. phureja & $0.6-42.7$ & crude, freeze-dried & (Griffiths et al., 2007) \\
\hline S. tuberosum & $1.3-58$ & $\begin{array}{l}\text { cooked, without skin, } \\
\text { mashed }\end{array}$ & (Kaspar et al., 2011) \\
\hline Solanum sp. & $1.7-84.2$ & crude & (Brown et al., 2007) \\
\hline S. tuberosum & $2.6-14.8$ & crude, freeze-dried & (Burmeister et al., 2011) \\
\hline S. phureja & $8.9-9.9$ & crude, freeze-dried & (Burmeister et al., 2011) \\
\hline S. phureja & $4.9-92$ & crude & (Burgos et al., 2009b) \\
\hline S. tuberosum & $4.8-46.5$ & crude & (Reddivari et al., 2007) \\
\hline S. phureja & $1.0-21.4$ & crude, freeze-dried & (Bonierbale et al., 2009) \\
\hline S. tuberosum & $1-3$ & crude, freeze-dried & (Leo et al., 2008) \\
\hline S. tuberosum & $2.8-36.2$ & crude, freeze-dried & (André et al., 2007a) \\
\hline
\end{tabular}

Table 5. Total carotenoid content in potatoes reported in literature. Contents reported in another unit than $\mu \mathrm{g} / \mathrm{g}$ dry weight (DW) were recalculated utilizing an average moisture content of $80 \%$. 


\begin{tabular}{|c|c|c|c|}
\hline Plant material & $\begin{array}{c}\text { Carotenoid content } \\
(\mu \mathrm{g} / \mathrm{g} D W)\end{array}$ & $\begin{array}{c}\text { Sample } \\
\text { preparation }\end{array}$ & Reference \\
\hline \multicolumn{4}{|l|}{ Lutein } \\
\hline S. tuberosum & 0.5 & crude & $\begin{array}{lll}\text { (Blessington et al., } \\
2010)\end{array}$ \\
\hline S. tuberosum & $1.0-6.0$ & crude & $\begin{array}{l}\text { (Nesterenko \& Sink, } \\
\text { 2003) }\end{array}$ \\
\hline $\begin{array}{l}\text { S. tuberosum cv. } \\
\text { Yukon Gold and } \\
\text { Superior }\end{array}$ & 1.2 and 0.8 & $\begin{array}{l}\text { crude, without } \\
\text { skin }\end{array}$ & (Lu \& Haynes, 2001) \\
\hline $\begin{array}{l}\text { Hybrid from S. } \\
\text { phureja and } S . \\
\text { stenotomum }\end{array}$ & $2.8-26.6$ & $\begin{array}{l}\text { crude, without } \\
\text { skin }\end{array}$ & (Lu \& Haynes, 2001) \\
\hline $\begin{array}{l}\text { Cross between } S . \\
\text { tuberosum and } S . \\
\text { phureja }\end{array}$ & $0.8-11.6$ & & (Kobayashi et al., 2008) \\
\hline S. tuberosum & n.d. -0.03 & crude & (Zhou et al., 2011) \\
\hline $\begin{array}{l}\text { S. tuberosum cv. } \\
\text { Baltica }\end{array}$ & 3.3 & $\begin{array}{l}\text { cooked and } \\
\text { mashed }\end{array}$ & (Bub et al., 2008) \\
\hline $\begin{array}{l}\text { S. tuberosum cv. Red } \\
\text { Laura and Shetland } \\
\text { Black }\end{array}$ & 3.2 and 0.5 & $\begin{array}{l}\text { crude, freeze- } \\
\text { dried }\end{array}$ & $\begin{array}{l}\text { (Burmeister et al., } \\
\text { 2011) }\end{array}$ \\
\hline $\begin{array}{l}\text { S. phureja cv. Mayan } \\
\text { Twilight and Mayan } \\
\text { Gold }\end{array}$ & 2.1 and 2.7 & $\begin{array}{l}\text { crude, freeze- } \\
\text { dried }\end{array}$ & $\begin{array}{l}\text { (Burmeister et al., } \\
\text { 2011) }\end{array}$ \\
\hline S. phureja & $2.8-10.6$ & crude & (Burgos et al., 2009b) \\
\hline $\begin{array}{l}\text { S. tuberosum cv. } \\
\text { Désirée }\end{array}$ & 0.7 & $\begin{array}{l}\text { crude, freeze- } \\
\text { dried }\end{array}$ & (Ducreux et al., 2005) \\
\hline S. tuberosum & $1.1-17.7$ & $\begin{array}{l}\text { crude, freeze- } \\
\text { dried }\end{array}$ & (André et al., 2007b) \\
\hline S. phureja & $0.6-1.9$ & $\begin{array}{l}\text { crude, freeze- } \\
\text { dried }\end{array}$ & (Bonierbale et al., 2009) \\
\hline S. tuberosum & $5.0-11.4$ & $\begin{array}{l}\text { crude, freeze- } \\
\text { dried }\end{array}$ & (André et al., 2009b) \\
\hline \multicolumn{4}{|l|}{ Violaxanthin } \\
\hline S. tuberosum & $0.2-6.2$ & crude & $\begin{array}{l}\text { (Nesterenko \& Sink, } \\
\text { 2003) }\end{array}$ \\
\hline $\begin{array}{l}\text { S. tuberosum cv. } \\
\text { Yukon Gold and } \\
\text { Superior }\end{array}$ & 2.6 and 1.0 & $\begin{array}{l}\text { crude, without } \\
\text { skin }\end{array}$ & (Lu \& Haynes, 2001) \\
\hline $\begin{array}{l}\text { Hybrid from S. } \\
\text { phureja and S. } \\
\text { stenotomum }\end{array}$ & $1.2-22.0$ & $\begin{array}{l}\text { crude, without } \\
\text { skin }\end{array}$ & (Lu \& Haynes, 2001) \\
\hline S. tuberosum & $0.2-3.3$ & $\begin{array}{l}\text { crude, without } \\
\text { skin }\end{array}$ & $\begin{array}{l}\text { (Breithaupt \& Bamedi, } \\
\text { 2002) }\end{array}$ \\
\hline S. tuberosum & n.d. -0.03 & crude & (Zhou et al., 2011) \\
\hline $\begin{array}{l}\text { S. tuberosum cv. Red } \\
\text { Laura and Shetland }\end{array}$ & 6.1 and 1.3 (9-cis) & $\begin{array}{l}\text { crude, freeze- } \\
\text { dried }\end{array}$ & $\begin{array}{l}\text { (Burmeister et al., } \\
\text { 2011) }\end{array}$ \\
\hline
\end{tabular}




\begin{tabular}{|c|c|c|c|}
\hline Plant material & $\begin{array}{c}\text { Carotenoid content } \\
(\mu \mathrm{g} / \mathrm{g} \text { DW })\end{array}$ & $\begin{array}{c}\text { Sample } \\
\text { preparation }\end{array}$ & Reference \\
\hline \multicolumn{4}{|l|}{ Black } \\
\hline S. phureja cv. Mayan & 2.9 and 3.1 (9-cis) & crude, freeze- & (Burmeister et al., \\
\hline Twilight and Mayan & & dried & 2011) \\
\hline \multicolumn{4}{|l|}{ Gold } \\
\hline S. phureja & traces -20.5 & crude & (Burgos et al., 2009b) \\
\hline $\begin{array}{l}\text { S. tuberosum cv. } \\
\text { Désirée }\end{array}$ & 2.2 & $\begin{array}{l}\text { crude, freeze- } \\
\text { dried }\end{array}$ & (Ducreux et al., 2005) \\
\hline S. phureja cv. Mayan & 11.7 & crude, freeze- & (Ducreux et al., 2005) \\
\hline Gold & & dried & \\
\hline \multicolumn{4}{|l|}{ Zeaxanthin } \\
\hline $\begin{array}{l}\text { Cross between } S \text {. } \\
\text { tuberosum and } S \text {. } \\
\text { phureja }\end{array}$ & $7.7-24.6$ & & (Kobayashi et al., 2008) \\
\hline S. tuberosum & n.d. -0.5 & crude & (Zhou et al., 2011) \\
\hline $\begin{array}{l}\text { S. tuberosum cv. } \\
\text { Baltica }\end{array}$ & 0.6 & $\begin{array}{l}\text { cooked and } \\
\text { mashed }\end{array}$ & (Bub et al., 2008) \\
\hline S. phureja & traces -64.5 & crude & (Burgos et al., 2009b) \\
\hline S. tuberosum & $0-17.7$ & $\begin{array}{l}\text { crude, freeze- } \\
\text { dried }\end{array}$ & (André et al., 2007b) \\
\hline S. tuberosum & $2.7-4.3$ & $\begin{array}{l}\text { crude, freeze- } \\
\text { dried }\end{array}$ & (André et al., 2009b) \\
\hline \multicolumn{4}{|l|}{ Antheraxanthin } \\
\hline S. tuberosum & $0.4-2.4$ & $\begin{array}{l}\text { crude, without } \\
\text { skin }\end{array}$ & $\begin{array}{l}\text { (Breithaupt \& Bamedi, } \\
\text { 2002) }\end{array}$ \\
\hline S. phureja & $0.3-18.8$ & crude & (Burgos et al., 2009b) \\
\hline S. phureja cv. Mayan & 4.2 & crude, freeze- & (Ducreux et al., 2005) \\
\hline Gold & & dried & \\
\hline S. phureja & $0.03-3.54$ & $\begin{array}{l}\text { crude, freeze- } \\
\text { dried }\end{array}$ & (Bonierbale et al., 2009) \\
\hline \multicolumn{4}{|c|}{ Lutein-5,6-epoxide (Taraxanthin) } \\
\hline S. tuberosum cv. & 0.9 and 0.5 & crude, without & (Lu \& Haynes, 2001) \\
\hline Yukon Gold and & & skin & \\
\hline Superior & & & \\
\hline Hybrid from $S$. & $1.1-27.4$ & crude, without & (Lu \& Haynes, 2001) \\
\hline $\begin{array}{l}\text { phureja and S. } \\
\text { stenotomum }\end{array}$ & & skin & \\
\hline \multicolumn{4}{|l|}{$\beta$-Carotene } \\
\hline S. tuberosum cv. & 0.7 & cooked and & (Bub et al., 2008) \\
\hline Baltica & & mashed & \\
\hline S. phureja & $0-1.4$ & crude & (Burgos et al., 2009b) \\
\hline S. tuberosum & $0-2.2$ & $\begin{array}{l}\text { crude, freeze- } \\
\text { dried }\end{array}$ & (André et al., 2007b) \\
\hline
\end{tabular}

Table 6. Individual carotenoid content of potatoes reported in literature. Contents reported in another unit than $\mu \mathrm{g} / \mathrm{g}$ dry weight $(\mathrm{DW})$ were recalculated utilizing an average moisture content of $80 \%$. 
Total carotenoid contents in potatoes were reported to be between 0.6 and $233 \mu \mathrm{g} / \mathrm{g}$ DW. None of the carotenoids were quantifiable in all potatoes under study and a huge range of the contents of the individual compounds was observed. In potatoes where lutein was quantifiable, the contents ranged between 0.5 and $26.6 \mu \mathrm{g} / \mathrm{g}$ DW. The contents of violaxanthin, zeaxanthin, antheraxanthin, lutein-5,6-epoxide, and $\beta$-carotene ranged between 0.2 and $22.0 \mu \mathrm{g} / \mathrm{g} \mathrm{DW}, 0.6$ and $64.5 \mu \mathrm{g} / \mathrm{g}$ DW, 0.03 and $18.8 \mu \mathrm{g} / \mathrm{g}$ DW, 0.5 and 27.4 $\mu \mathrm{g} / \mathrm{g}$ DW, and 0.7 and $2.2 \mu \mathrm{g} / \mathrm{g} \mathrm{DW}$, respectively.

\subsection{Ascorbic acid}

Regarding the content of ascorbic acid (vitamin C, chemical structure is shown in Figure 3) in potatoes, a huge number of publications are available. Quantification was done either by HPLC-UV, a spectrophotometric assay (after addition of 2,6-dichloroindophenol or 2,4dinitrophenylhydrazine) or by fluorescence measurement (after addition of sodium acetate and O-phenylene diamine, for references see Table 7). In most cases, the total ascorbic acid content including dihydroascorbic acid was determined. Table 7 shows the contents of ascorbic acid that have been described in literature. The amounts ranged from 0.2 to 5.6 $\mathrm{mg} / \mathrm{g}$ DW.<smiles>O=C1O[C@H]([C@H](O)CO)C(O)=C1O</smiles>

Fig. 3. Chemical structure of ascorbic acid (vitamin C).

\subsection{Vitamin E}

In addition to the antioxidants described above, potatoes also contain vitamin E. Vitamin $\mathrm{E}$ includes four tocopherols ( $\alpha, \beta, \gamma$, and $\delta$ ) and four tocotrienols $(\alpha, \beta, \gamma$, and $\delta)$, among which $\alpha$-tocopherol has the highest biological activity in humans. In potatoes, $\alpha$-tocopherol is the predominant vitamin $E$ representative. $\gamma$-Tocopherol and a-tocotrienol were detectable in minor amounts (André et al., 2007b, Chun et al., 2006, Crowell et al., 2008). The amounts of a-tocopherol found in potatoes are summarized in Table 8 . When recalculated to the DW of potatoes, the amounts ranged between 0.8 and $34.8 \mu \mathrm{g} / \mathrm{g}$ DW.

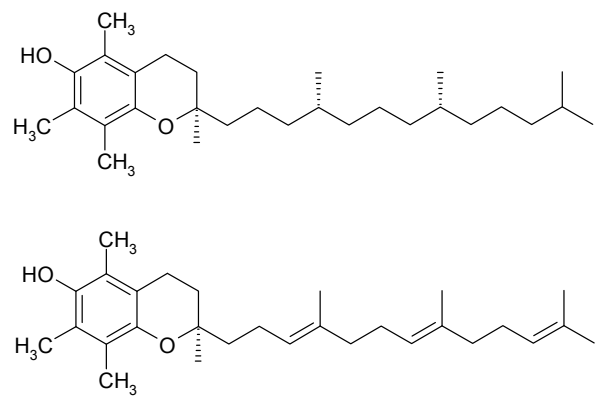

Fig. 4. Chemical structures of the vitamin E representatives a-tocopherol (above) and atocotrienol (below). 


\begin{tabular}{|c|c|c|c|}
\hline Plant material & $\begin{array}{c}\text { Ascorbic acid } \\
\text { content }(\mathrm{mg} / \mathrm{g} \text { DW) }\end{array}$ & $\begin{array}{c}\text { Sample } \\
\text { preparation }\end{array}$ & Reference \\
\hline S. tuberosum & 1.4 & Skin, crude & (Singh et al., 2011) \\
\hline S. tuberosum & $0.5-1.7$ & crude, freeze-dried & (Dale et al., 2003) \\
\hline $\begin{array}{l}\text { S. tuberosum L. cv. } \\
\text { Favorite }\end{array}$ & 4.2 & crude & (Qin et al., 2011) \\
\hline $\begin{array}{l}\text { S. tuberosum cvs. Bintje, } \\
\text { Piccolo and Purple } \\
\text { Majesty }\end{array}$ & $0.4-0.6$ & crude, freeze-dried & (Navarre et al., 2010) \\
\hline $\begin{array}{l}\text { S. tuberosum ssp. } \\
\text { Andigenum }\end{array}$ & $0.4-0.7$ & crude & (Jimenez et al., 2009) \\
\hline S. tuberosum cv. Spunta & 1.4 & crude & (Jimenez et al., 2009) \\
\hline S. tuberosum & $0.6-1.5$ & crude, freeze-dried & (Love \& Salaiz, 2004) \\
\hline $\begin{array}{l}\text { S. tuberosum cvs. Russet } \\
\text { Burbank and Shepody }\end{array}$ & $0.5-1.2$ & crude, freeze-dried & (Rogan et al., 2000) \\
\hline $\begin{array}{l}\text { S. tuberosum cv. } \\
\text { Norkotah and Ranger }\end{array}$ & 0.4 and 0.8 & crude, freeze-dried & $\begin{array}{l}\text { (Shakya \& Navarre, } \\
\text { 2006) }\end{array}$ \\
\hline S. tuberosum & $0.3-0.7$ & crude & (Hajslova et al., 2005) \\
\hline $\begin{array}{l}\text { S. tuberosum and S. } x \\
\text { chaucha }\end{array}$ & $2.0-5.6$ & crude & $\begin{array}{l}\text { (Rodriguez et al., } \\
\text { 2010) }\end{array}$ \\
\hline S. tuberosum & $0.5-1.0$ & crude, freeze-dried & (Leo et al., 2008) \\
\hline S. tuberosum & $0.8-2.1$ & crude & (Han et al., 2004) \\
\hline S. tuberosum & 1.9 & crude & (Natella et al., 2010) \\
\hline S. tuberosum & $0.2-1.2$ & crude & (Burgos et al., 2009a) \\
\hline S. tuberosum & $0.2-1.8$ & crude, freeze-dried & $\begin{array}{l}\text { (André et al., 2007a, } \\
\text { André et al., 2009b) }\end{array}$ \\
\hline
\end{tabular}

Table 7. Ascorbic acid content in potatoes reported in literature. Contents reported in another unit than $\mathrm{mg} / \mathrm{g}$ dry weight (DW) were recalculated utilizing an average moisture content of $80 \%$.

\begin{tabular}{|c|c|c|c|}
\hline Plant material & $\begin{array}{c}\text { a-Tocopherol } \\
\text { content }(\mu \mathrm{g} / \mathrm{g} \text { DW) }\end{array}$ & $\begin{array}{c}\text { Sample } \\
\text { preparation }\end{array}$ & Reference \\
\hline S. tuberosum cv. Spunta & 2.1 & crude & (Crowell et al., 2008) \\
\hline S. tuberosum cv. Spunta & 1.4 & crude & (Crowell et al., 2008) \\
\hline S. tuberosum & $2.7-34.8$ & $\begin{array}{l}\text { Andean cultivars, } \\
\text { crude, freeze-dried }\end{array}$ & $\begin{array}{l}\text { (André et al., 2007b, } \\
\text { André et al., 2009b) }\end{array}$ \\
\hline S. tuberosum cv. Nicola & 0.8 & crude, freeze-dried & (André et al., 2007b) \\
\hline $\begin{array}{l}\text { S. tuberosum cv. } \\
\text { Vitelotte }\end{array}$ & 2.3 & crude, freeze-dried & (André et al., 2007b) \\
\hline S. tuberosum & 3.5 & crude & (Chun et al., 2006) \\
\hline S. tuberosum & 3.0 & boiled & (Chun et al., 2006) \\
\hline S. tuberosum & $0.7-1.4$ & stored, crude & $\begin{array}{l}\text { (Spychalla \& } \\
\text { Desborough, 1990) }\end{array}$ \\
\hline S. tuberosum cv. Désirée & 22.5 & crude, freeze-dried & (Ducreux et al., 2005) \\
\hline
\end{tabular}

Table 8. Vitamin E (a-tocopherol) content in potatoes reported in literature. Contents reported in another unit than $\mu \mathrm{g} / \mathrm{g}$ dry weight (DW) were recalculated utilizing an average moisture content of $80 \%$. 


\section{Impact of cultivation, storage and processing on antioxidant compounds in potato}

\subsection{Impact of cultivation conditions}

Environmental or abiotic stresses may have an impact on antioxidant contents, as these can generate reactive oxygen species in plants, thereby causing oxidative stress and impacting the antioxidant responses. Many plant secondary metabolites are determinants of both plant stress tolerance and nutritional quality (Jansen et al., 2008). In a study on potato exposed to drought stress (André et al., 2009c), highly cultivar-specific responses were observed. Thus, in yellow tubers, changes in the contents of antioxidants were weak, whereas in pigmented (red- and purple fleshed) tubers, variations (reduction or increase) were high. Hamouz et al. (2010) reported higher total polyphenol contents in potatoes grown under drought in a warm lowland location and under low temperatures during the vegetation period, both reflecting extreme climatic conditions. Carotenoid contents increased in most of the cases, whereas vitamin $C$ was not affected by drought, except in one of the investigated cultivars. In a review on plant stress and human health (Jansen et al., 2008), polyphenol and vitamin C changes upon exposure of a range of plants to UV-B radiation were variable and dependent on the experimental system under investigation. It can be said that the effect of a stress on the nutritional composition of plants is still poorly understood and needs further investigations.

Higher anthocyanins and total phenolics were observed at locations with longer days and cooler temperatures (Reyes et al., 2004); according to the authors, light and day length may have an influence on the phenylpropanoid metabolism thereby affecting/favouring the synthesis of some compounds. Lachman et al. (2008) compared total phenolic contents from potato tubers grown in 4 different localities and attributed higher total phenolic contents (a difference of approximately $12 \%$ between highest and lowest contents) at harvest mainly to lower temperatures in the end of the vegetation period.

Concerning cultivation methods (conventional versus organic), the nutrient composition of plants is affected by the differences in organic and conventional farming, mainly due to fertilization management (Soltoft et al., 2010). However, it cannot clearly be said that significant differences in health-promoting compounds exist between the two farming systems. Potatoes produced in organic cultivation when compared to conventional cultivation contain higher levels of chlorogenic acid (Hajslova et al., 2005, Soltoft et al., 2010). Vitamin $C$ content in the tubers from organically grown potatoes were not significantly higher than those from conventional farming (Hajslova et al., 2005, Rembialkowska, 1998). More generally, Faller and Fialho (2010) found that polyphenol content and antioxidant capacity in plant foods (several vegetables and fruits) were mostly similar or slightly higher in organic agriculture. Further studies are however necessary to clarify the impact of organic versus conventional agriculture on the contents of antioxidants in potato.

\subsection{Post-harvest storage}

Potatoes are mainly grown as single crop, with subsequent storage for three to ten months, a period in which they may undergo high metabolic activities (Pinhero et al., 2009). 
It is known that ascorbic acid in fresh foods, including potato, is not stable postharvest (Pinhero et al., 2009) with levels decreasing to 40 to $60 \%$ of the initial value (Woolfe, 1987) or even to about $25 \%$ of the original one (Dale et al., 2003). Similarly, according to Burgos et al. (2009a), ascorbic acid concentrations decreased as the storage time increased.

As for carotenoids, Griffiths et al. (2007) found that postharvest storage reduces carotenoid contents, whereas Morris et al. (2004) describe only slight or no total carotenoid content changes at all during storage at cold temperatures. However, according to Morris et al. (2004), individual carotenoid levels do change during storage. Blessington et al. (2010) describe higher carotenoid contents (contents increase between 1 and $100 \%)$ in stored $\left(4^{\circ} \mathrm{C}\right.$ and $20^{\circ} \mathrm{C}$ ) potatoes as compared to non-stored potatoes.

Storage at $4^{\circ} \mathrm{C}$ and $20^{\circ} \mathrm{C}$ also increased the total phenolic contents compared to non-stored potatoes (Blessington et al., 2010).

According to Dale et al. (2003), storage would be the major effect impacting contents of antioxidants, whereas location, year and genotype also would play a role. Interestingly for vitamin $\mathrm{C}$, there is a high level of consistency in the ranking of genotypes across years, indicating heritability, a trait to be exploited during breeding. Burgos et al. (2009a) corroborate this information and found that the genotype effect is higher than the environment and than the genotype $x$ environment interaction.

\subsection{Cooking}

Vitamin C changes during cooking have been evaluated by Burgos et al. (2009a) by boiling, microwaving and baking tubers. Losses of vitamin $C$ were most important in baked and microwaved tubers as compared to boiled tubers. The percentage of retention ranged from 53 to $97 \%$, from 6 to $66 \%$ and from 6 to 39\% in boiled, baked and microwaved potatoes. In new potatoes, harvested at a young developmental stage, contents of total phenolics and vitamin $C$ did not decrease after cooking by any method, presumably because of the small size of these tubers and the short cooking time required (Navarre et al., 2010).

Similarly, according to $\mathrm{Xu}$ et al. (2009), antioxidant capacity, mainly determined by the potato variety, was slightly influenced by cooking conditions depending on the cultivar. According to Blessington et al. (2010), when comparing different cooking methods of potatoes prepared with skin (baking, boiling, frying, microwaving), boiled samples were lower in total carotenoid contents, whereas for total phenolics, increased contents were observed after baking, frying and microwaving as compared to uncooked samples. Mulinacci et al. (2008) describe unaltered phenolic acid contents after cooking and microwaving unpeeled potatoes; however, in coloured potatoes, a decrease in the total anthocyanin content was observed (decrease in the range 16-29\%). It has been shown by Dao and Friedman (1992) that peeling influences the contents of health-promoting compounds; they showed that potato peels contain high levels of phenolics, making them a promising material for the generation of functional foods. Mattila and Hellström (2007) observed a decrease in phenolics when comparing a peeled and cooked potato with an uncooked potato. It was also suggested that during the cooking process (cooking with skin), phenolics might migrate from the peel into the cortex and the internal tissues suggesting an improved extractability from cooked samples. 
A more specific processing is the so-called chuno production. Chuno corresponds to a traditional Andean freeze and sun-dried potato, in which water is completely removed by mechanical pressing facilitated through freeze and thaw cycles implicating destruction of cellular structure (Penarrieta et al., 2011). During this process, a loss of antioxidants and phenolic compounds was shown, though some compounds seemed to be transferred from the peel to the flesh during the process, as also reflected by the dark to black colour of the chuno.

\section{Nutritional relevance and health-beneficial properties of potato antioxidants}

Oxidative stress is a disturbance of the equilibrium between pro-oxidants and antioxidants, in favour of the former (Kaspar et al., 2011). This imbalance may lead to cellular damage, as oxidation of cellular lipids, proteins and DNA imparts cellular function and increases susceptibility to a number of chronic diseases. Antioxidant molecules may scavenge reactive oxygen species, thereby limiting oxidative stress (Robert et al., 2006).

\subsection{Vitamins}

Potato is known to be a good source of vitamin C in the human diet. In the human body, ascorbate plays a role as a water-soluble antioxidant and as cofactor in reactions catalyzed by a number of metal-dependent oxygenases (André et al., 2010). Besides its positive effect on human health, it is also important to mention that vitamin C plays an important role as enhancer of iron bioavailability from potato (Yun et al., 2004).

As for vitamin E, its major biological role is to prevent lipid peroxidation and to protect poly unsaturated fatty acids and low density lipoproteins from oxidation by free radicals (André et al., 2010).

\subsection{Carotenoids and polyphenols}

As previously said, potatoes are good sources of antioxidants, such as the hydrophilic polyphenols and only moderate sources of the lipophilic carotenoids. Dietary carotenoids are associated with health benefits. On one hand, the provitamin A activity of carotenoids, such as beta-carotene, alpha-carotene and beta-cryptoxanthin is well-known; on the other hand, non pro-vitamin A carotenoids, such as lutein and zeaxanthin for example, have important antioxidant activity and are known to provide protection against age-related macular degeneration (Griffiths et al., 2007).

A correlation between polyphenol intake and reduced incidence of cancers, cardiovascular and neurodegenerative diseases was shown by Arts and Hollman (2005); however these positive effects could not only be attributed to their antioxidant properties. Polyphenols also exert their health beneficial effects through modulation of cellular signalling processes: as inflammation modulatory agents, as regulators of cell proliferation and differentiation, angiogenesis and apoptosis and as modulators of signalling cascades and apoptotic processes (reviewed in (Stevenson \& Hurst, 2007)).

Few studies are available in the literature on health-promoting effects of potato antioxidants and will be presented hereafter. The impact of the consumption of pigmented potatoes on 
oxidative stress and inflammatory damage in man has been studied by Kaspar et al. (2011). Men consumed either white, yellow (high concentrations in phenolic acis and carotenoids) or purple fleshed (high concentrations of phenolic acids and anthocyanins) potato once per day in a randomized 6-week study with good compliance. The consumption of pigmented potato resulted in elevated antioxidant status and reduced inflammation and DNA damage, as reflected by e.g. decreased inflammatory cytokine and C-reactive protein concentrations. Another study on the lipid-lowering effect of potato in rats showed that feeding rats a potato-enriched diet led to a decrease in cholesterol and triglyceride levels in plasma, decreased cholesterol level in the liver and improved antioxidant status (Robert et al., 2006, Robert et al., 2008). These results suggest that potato consumption may enhance antioxidant defense and improve the lipid metabolism. Similarly in a study performed by Han et al. (2006) on rats fed with anthocyanin-rich purple potato flake extracts, it was shown that these extracts have antioxidant capacity with regard to radical scavenging activity and inhibition of linoleic acid oxidation; moreover, they would enhance hepatic Mn-SOD, Cu/Zn-SOD and GSH-Px mRNA expression suggesting a reduced hepatic lipid peroxidation and an improved antioxidant potential in the rats.

In a study performed by Thompson et al. (2009) on induced breast cancer in rats, a greater inhibition of carcinogenesis was shown when the rats were fed with a red pigmented cultivar as compared to a White Russet Burbank. The red cultivar had high levels of anthocyanin and chlorogenic acid derivatives, previously reported to inhibit the growth of human breast cancer cells grown in monolayer culture (Hakimuddin et al., 2004).

\section{Bioavailability}

Studies on the bioavailability of antioxidants and/or clinical outcomes following the administration of antioxidants from potato are scarce. Concerning nutrition, the term bioavailability describes the quantity of an ingested nutrient that is used by the body in its original or metabolized form. Many problems are to be faced, such as those related to cultivar variability, sample preparation and metabolism in the organism as well as interaction with gut microflora.

A few studies on the bioavailability of $\beta$-carotene from sweet potato are available (Bengtsson et al., 2009, Failla et al., 2009). It is worth mentioning that the bioavailability of carotenoids is dependent on a number of parameters including the physicochemical state, cooking style, other components of the meal (e.g. fat content), to name only a few. Failla et al. (2009) pointed to a relatively poor bioaccessibility (i.e. the release from the food matrix and the solubility in the gastro-intestinal fluids) of $\beta$-carotene from sweet potato together with a poor micellarization; this latter process corresponds to the transfer of the carotenoids from the food matrix into mixed bile salt micelles, during the small intestinal phase of digestion, a process that was however improved by the addition of oil. In a study performed by Bub et al. (2008) on genetically modified, zeaxanthin-enriched potato ( $270 \mu \mathrm{g} / 100 \mathrm{~g})$, the concentration of zeaxanthin was significantly increased in chylomicrons, a group of lipoproteins reflecting newly absorbed carotenoids, after the consumption of genetically modified potatoes (by three men, randomized, controlled double-blinded) as compared to no increase after consumption of control potatoes $(12.9 \mu \mathrm{g} / 100 \mathrm{~g})$. 
There are a number of studies on the bioavailability of polyphenols, such as e.g. D'Archivio et al. (2010), Manach et al. (2004), Scalbert and Williamson (2000), and Williamson and Manach (2005), though none specifically on potatoes. Within polyphenols, the bioavailability greatly differs and decreases from isoflavones, to flavonols, to flavan-3-ols, to anthocyanins and proanthocyanidins. The difficulty in realizing polyphenol biovailability studies lies in the fact that they may undergo substantial modifications following ingestion. Some compounds may be absorbed in the small intestine. Others reach the colon where they undergo modifications such as hydrolization of glycosides into aglycones by the colonic microflora. Prior to the passage into the blood stream, other modifications might occur including methylation, sulfation, glucuronidation (D'Archivio et al., 2010). Since chlorogenic acid is the major phenolic acid present in potato, it is worth mentioning a study on the bioavailability of chlorogenic acid from coffee (Stalmach et al., 2010). This study implicating human ileostomy volunteers indicates a potential absorption of $29 \%$ of the intake by the small intestine; thus approximately one third of ingested chlorogenic acid in foods would be absorbed and enter the bloodstream; in healthy subjects with a functioning colon, the remaining part would reach the large intestine. A recent publication on the bioavailability of quercetin 3-O-rutinoside included healthy volunteers and those with an ileostomy. After consumption of tomato juice fortified with quercetin 3-O-rutinoside, no metabolites were detected in the plasma and urine of the ileostomists and $86 \%$ of the ingested quercetin 3-Orutinoside was detected in the ileostomy bag. In healthy subjects, this amount reaches the colon. In the colon, the quercetin 3-O-rutinoside is converted to phenolic acids by the microflora. Amounts of these phenolic acids corresponding to $22 \%$ of the quercetin $3-O-$ rutinoside intake have been detected in the urine of the healthy volunteers (Jaganath et al., 2006).

Informations on the bioavailability of a-tocopherol are scarce. An in vitro study with broccoli showed that $20 \%$ of the applied a-tocopherol has been incorporated into the aquaeous phase by micellarization in a digestion model (Granado et al., 2006). The bioavailability of atocopherol in humans was assessed after the consumption of $\mathrm{d}_{6}$ - $\mathrm{a}$-tocopherol spiked apples. When the apples were consumed together with a breakfast containing no fat, $10 \%$ of the 22 $\mathrm{mg} \mathrm{d}_{6^{-}} \mathrm{a}$-tocopherol were detected in the plasma of the probands. Increasing the breakfast fat content to $6 \%$ and $21 \%, 20 \%$ and $33 \%$ of the $d_{6-}$ a-tocopherol has been detected, respectively (Bruno et al., 2006).

The determination of the bioavailability of ascorbic acid in doses present in food is difficult. The plasma levels of healthy persons after oral ingestion of vitamin $C$ are saturated at 70 to $80 \mu \mathrm{M}$ (Duconge et al., 2008) and the ingestion of additional ascorbic acid does not lead to a simple increase of the plasma levels. A study with seven healthy vitamin C depleted volunteers showed that the bioavailability was $100 \%$ at doses of $200 \mathrm{mg}$. When using higher doses, the bioavailability was lower than $50 \%$ (Levine et al., 1996). When stable, isotopically labelled ascorbic acid was administered to four healthy probands, the unspecific measurement of ascorbic acid in their plasma revealed a $12 \%$ increase of the plasma level. When the samples were analysed for their isotope content, an increase of 3 to $6 \%$ was observed. The authors of the study stated that the ingested vitamin $\mathrm{C}$ dose enters an existing pool in the body, but this pool is continuously fluxed with vitamin $C$ present in the body before the beginning of the study (Bluck et al., 2005). Ascorbic acid bioavailability after ingestion of a fortified beverage and orange juice was approximately $65 \%$, but the standard deviations in this study have been quite high (Carter et al., 2010). 


\section{References}

André, C., Larondelle, Y., \& Evers, D. (2010). Dietary antioxidants and oxidative stress from a human and plant perspective: a review. Curr Nutr Food Sci. 6, 2-12

André, C. M., Ghislain, M., Bertin, P., Oufir, M., Herrera, M. R., Hoffmann, L., Hausman, J. F., Larondelle, Y., \& Evers, D. (2007a). Andean potato cultivars (Solanum tuberosum L.) as a source of antioxidant and mineral micronutrients. J Agric Food Chem. 55, 2, 366-378

André, C. M., Oufir, M., Guignard, C., Hoffmann, L., Hausman, J. F., Evers, D., \& Larondelle, Y. (2007b). Antioxidant profiling of native Andean potato tubers (Solanum tuberosum L.) reveals cultivars with high levels of beta-carotene, alphatocopherol, chlorogenic acid, and petanin. J Agric Food Chem. 55, 26, 10839-10849

André, C. M., Oufir, M., Hoffmann, L., Hausman, J. F., Rogez, H., Larondelle, Y., \& Evers, D. (2009a). Influence of environment and genotype on polyphenol compounds and in vitro antioxidant capacity of native Andean potatoes (Solanum tuberosum L.). J Food Compos Anal. 22, 517-524

André, C. M., Schafleitner, R., Guignard, C., Oufir, M., Aliaga, C. A., Nomberto, G., Hoffmann, L., Hausman, J. F., Evers, D., \& Larondelle, Y. (2009b). Modification of the health-promoting value of potato tubers field grown under drought stress: emphasis on dietary antioxidant and glycoalkaloid contents in five native andean cultivars (Solanum tuberosum L.). J Agric Food Chem. 57, 2, 599-609

André, C. M., Schafleitner, R., Legay, S., Lefevre, I., Aliaga, C. A., Nomberto, G., Hoffmann, L., Hausman, J. F., Larondelle, Y., \& Evers, D. (2009c). Gene expression changes related to the production of phenolic compounds in potato tubers grown under drought stress. Phytochemistry. 70, 9, 1107-1116

Arts, I. C. \& Hollman, P. C. (2005). Polyphenols and disease risk in epidemiologic studies. Am J Clin Nutr. 81, 1 Suppl, 317S-325S

Bengtsson, A., Larsson, A. M., \& Svanberg, U. (2009). In vitro bioaccessibility of betacarotene from heat-processed orange-fleshed sweet potato. J Agric Food Chem. 57, 20, 9693-9698

Blessington, T., Nzaramba, M. N., Scheuring, D. C., Hale, A. L., Reddivari, L., \& Miller, J. C., Jr. (2010). Cooking methods and storage treatments of potato: Effects on carotenoids, antioxidant activity, and phenolics. Am J Pot Res. 87, 479-491

Bluck, L. J. C., Jones, K. S., Coward, W. A., \& Bates, C. J. (2005). The "anomalous" absorption of labelled and unlabelled vitamin c in men. Br J Nutr. 93, 627-632

Bonierbale, M., Grüneberg, W., Amoros, W., Burgos, G., Salas, E., Porras, E., \& zum Felde, T. (2009). Total and individual carotenoid profiles in Solanum phureja cultivated potatoes: II. Development and application of near-infrared reflectance spectroscopy (NIRS) calibrations for germplasm characterization. J Food Compos Anal. 22, 509-516

Breithaupt, D. E. \& Bamedi, A. (2002). Carotenoids and carotenoid esters in potatoes (Solanum tuberosum L.): New insights into an ancient vegetable. J Agric Food Chem. 50, 7175-7181

Brown, C. R. (2005). Antioxidants in potato. Am J Potato Re. 82, 163-172

Brown, C. R. (2008). Breeding for phytonutrient enhancement of potato. Am J Pot Res. 85, 298-307 
Brown, C. R., Culley, D., Bonierbale, M., \& Amoros, W. (2007). Anthocyanin, carotenoid content, and antioxidant values in native south american potato cultivars. Hortscience. 42, 7, 1733-1736

Bruno, R. S., Leonard, S. W., Park, S., Zhao, Y., \& Traber, M. G. (2006). Human vitamin E requirements assessed with the use of apples fortified with deuterium-labeled $\alpha$ tocopheryl acetate. Am J Clin Nutr. 83, 299-304

Bub, A., Mösender, J., Wenzel, G., Rechkemmer, G., \& Briviba, K. (2008). Zeaxanthin is bioavailable from genetically modified zeaxanthin-rich potatoes. Eur J Nutr. 47, 99103

Burgos, G., Auqui, S., Amoros, W., Salas, E., \& Bonierbale, M. (2009a). Ascorbic acid concentration of native Andean potato varieties as affected by environment, cooking and storage. J Food Compos Anal. 22, 533-538

Burgos, G., Salas, E., Amoros, W., Auqui, M., Munoa, L., Kimura, M., \& Bonbierale, M. (2009b). Total and individual carotenoid profiles in Solanum phureja of cultivated potatoes: I. Concentrations and relationships as determined by spectrophotometry and HPLC. J Food Compos Anal. 22, 503-508

Burlingame, B., Mouillé, B., \& Charrondière, R. (2009). Nutrients, bioactive non-nutrients and anti-nutrients in potatoes. J Food Compos Anal. 22, 494-502

Burmeister, A., Bondiek, S., Apel, L., Kühne, C., Hillebrand, S., \& Fleischmann, P. (2011). Comparison of carotenoid and anthocyanin profiles of raw and boiled Solanum tuberosum and Solanum phureja tubers. $J$ Food Compos Anal. doi:10.1016/j.jfca.2011.03.006,

Campos, D., Noratto, G., Chirinos, R., Arbizu, C., Roca, W., \& Cisneros-Zevallos, L. (2006). Antioxidant capacity and secondary metabolites in four species of Andean tuber crops: native potato (Solanum sp.), mashua (Tropaeolum tuberosum Ruiz \& Pavón), Oca (Oxalis tuberosa Molina) and ulluco (Ullucus tuberosus Caldas). J Sci Food Agric. 86, 1481-1488

Carter, B., Monsivais, P., \& Drewnowski, A. (2010). Absorption of folic acid and ascorbic acid from nutrient comparable beverages. J Food Sci. 75, 9, H289-H293

Chiou, A., Salta, F. N., Kalogeropoulos, N., Mylona, A., Ntalla, I., \& Andrikopoulos, N. K. (2007). Retention and distribution of polyphenols after pan-frying of French fries in oils enriched with olive leaf extract. J Food Sci. 72, 8, S574-S584

Chun, J., Lee, J., Ye, L., Exler, J., \& Eitenmiller, R. R. (2006). Tocopherol and tocotrienol contents of raw and processed fruits and vegetables in the United States diet. J Food Compos Anal. 19, 196-204

Crowell, E. F., McGrath, J. M., \& Douches, D. S. (2008). Accumulation of vitamin E in potato (Solanum tuberosum) tubers. Transgenic Res. 17, 2, 205-217

D'Archivio, M., Filesi, C., Vari, R., Scazzocchio, B., \& Masella, R. (2010). Bioavailability of the polyphenols: status and controversies. Int J Mol Sci. 11, 4, 1321-1342

Dale, M. F. B., Griffiths, D. W., \& Todd, D. T. (2003). Effects of genotype, environment, and postharvest storage on the total ascorbate content of potato (Solanum tuberosum) tubers. J Agric Food Chem. 51, 244-248

Dao, L. \& Friedman, M. (1992). Chlorogenic acid content of fresh and processed potatoes determined by ultraviolet spectrophotometry. J Agric Food Chem. 40, 2152-2156 
del Mar Verde Mendez, C., Rodriguez Delgado, M. A., Rodriguez Rodriguez, E. M., \& Diaz, R. C. (2004). Content of Free Phenolic Compounds in Cultivars of Potatoes Harvested in Tenerife (Canary Islands). J Agric Food Chem. 52, 1323-1327

Diretto, G., Al-Babili, S., Tavazza, R., Papacchioli, V., Beyer, P., \& Giuliano, G. (2007). Metabolic engineering of potato carotenoid content through tuber-specific overexpression of a bacterial mini-pathway. PLoS One. 2, 4, e350-1-e350-8

Duconge, J., Miranda-Massari, J. R., Gonzalez, M. J., Jackson, J. A., Warnock, W., \& Riordan, N. H. (2008). Pharmacokinetics of vitamin C: insights into the oral and intravenous administration of ascorbate. PRHSJ. 27, 1, 7-19

Ducreux, L. J. M., Morris, W. L., Hedley, P. E., Shepherd, T., Davies, H. V., Milliam, S., \& Taylor, M. A. (2005). Metabolic engineering of high carotenoid potato tubers containing enhanced levels of $\beta$-carotene and lutein. J Exp Bot. 56, 81-89

ETH Zürich (30-4-2009), Schweizer Nährwertdatenbank, In: SwissFIR, 18-7-2011, Available from

http://nwdb.ethz.ch:8080/nwdb/request?xml=MessageData\&xml=MetaData\&xsl $=$ SearchField\&lan $=$ de\&pageKey $=$ Start

Everette, J. D., Bryant, Q. M., Green, A. M., Abbey, Y. A., Wangila, G. W., \& Walker, R. B. (2010). Thorough study of reactivity of various compound classes toward the FolinCiocalteu reagent. J Agric Food Chem. 58, 14, 8139-8144

Failla, M. L., Thakkar, S. K., \& Kim, J. Y. (2009). In vitro bioaccessibility of beta-carotene in orange fleshed sweet potato (Ipomoea batatas, Lam.). J Agric Food Chem. 57, 22, 10922-10927

Faller, A. L. K. \& Fialho, E. (2010). Polyphenol content and antioxidant capacity in organic and conventional plant foods. J Food Compos Anal. 23, 561-568

FAO (2009), In: FAOSTAT, 10-5-2011, Available from http://faostat.fao.org

French Information Center on Food Quality (2008), French Food Composition Table, In: French Agency for food, environmental and occupational health safety, 18-7-2011, Available from http://www.afssa.fr/TableCIQUAL/

Giusti, M. M. and Wrolstad, R. E. (2001). Characterization and measurement of anthocyanins by UV-visible spectroscopy, In: Current Protocols in Food Analytical Chemistry, Wrolstad, R. E., Acree, T. E., Decker, E. A., Penner, M. H., Reid, D. S., Schwartz, S. J., Shoemaker, C. F., Smith, D. M., and Sporns, P., pp.(F1.2.1-F1.2.13), John Wiley \& Sons, Inc., 9780471142911, New York

Granado, F., Olmedilla, B., Herrero, C., Perez-Sacristan, B., Blanco, I., \& Blazquez, S. (2006). Bioavailability of carotenoids and tocopherols from broccoli: In vivo and in vitro assessment. Exp Biol Med. 231, 1733-1738

Griffiths, D. W., Dale, M. F. B., Morris, W. L., \& Ramsay, G. (2007). Effects of season and postharvest storage on the carotenoid content of Solanum phureja potato tubers. $J$ Agric Food Chem. 55, 379-385

Hajslova, J., Schulzova, V., Slanina, P., Janne, K., Hellenäs, K. E., \& Andersson, C. (2005). Quality of organically and conventionally grown potatoes: Four-year study of micronutrients, metals, secondary metabolites, enzymic browning and organoleptic properties. Food Addit Contam. 22, 6, 514-534

Hakimuddin, F., Paliyath, G., \& Meckling, K. (2004). Selective cytotoxicity of a red grape wine flavonoid fraction against MCF-7 cells. Breast Cancer Res Treat. 85, 1, 65-79 
Hamouz, K., Lachman, J., Hejtmankova, A., Pazderu, K., Cizek, M., \& Dvorak, O. (2010). Effect of natural and growing conditions on the content of phenolics in potatoes with different flesh colour. Plant Soil Environ. 56, 8, 368-374

Han, J.-S., Kozukue, N., Young, K.-S., Lee, K.-R., \& Friedman, M. (2004). Distribution of ascorbic acid in potato tubers and in home-processed and commercial potato foods. J Agric Food Chem. 52, 6516-6521

Han, K. H., Sekikawa, M., Shimada, K., Hashimoto, M., Hashimoto, N., Noda, T., Tanaka, H., \& Fukushima, M. (2006). Anthocyanin-rich purple potato flake extract has antioxidant capacity and improves antioxidant potential in rats. Br J Nutr. 96, 6, $1125-1133$

Hirschi, K. D. (2009). Nutrient Biofortification of food crops. Annu Rev Nutr. 29, 401-421

Huaman, Z. \& Spooner, D. M. (2002). Reclassification of landrace populations of cultivated potatoes (Solanum sect. Petota). Am J Bot. 89, 6, 947-965

Im, H. W., Suh, B. S., Lee, S. U., Kozukue, N., Ohnisi-Kameyama, M., Levin, C. E., \& Friedman, M. (2008). Analysis of phenolic compounds by high-performance liquid chromatography and liquid chromatography/mass spectrometry in potato plant flowers, leaves, stems, and tubers and in home-processed potatoes. J Agric Food Chem. 56, 9, 3341-3349

Jaganath, I. B., Mullen, W., Edwards, C. A., \& Crozier, A. (2006). The relative contribution of the small and large intestine to the absorption and metabolism of rutin in man. Free Radical Res. 40, 10, 1035-1046

Jansen, M. A. K., hectors, K., O-Brien, N. M., Guisez, Y., \& Potters, G. (2008). Plant stress and human health: do human consumers benefit from UV-B acclimated crops? Plant Science. 175, 449-458

Jimenez, M. E., Rossi, A. M., \& Samman, N. C. (2009). Phenotypic, agronomic and nutritional characteristics of seven varieties of Andean potatoes. J Food Compos Anal. 22, 613-616

Kaspar, K. L., Park, J. S., Brown, C. R., Mathison, B. D., Navarre, D. A., \& Chew, B. P. (2011). Pigmented potato consumption alters oxidative stress and inflammatory damage in men. J Nutr. 141, 1, 108-111

Kobayashi, A., Ohara-Takada, A., Tsuda, S., Matsuura-Endo, C., Takada, N., Umemura, Y., Nakao, T., Yoshida, T., Hayashi, K., \& Mori, M. (2008). Breeding of potato variety "Inca-no-hitomi" with a very high carotenoid content. Breeding Sci. 58, 77-82

Kotikova, Z., Hejtmankova, A., Lachman, J., Hamouz, K., Trnkova, E., \& Dvorak, P. (2007). Effect of selected factors on total carotenoid content in potato tubers (Solanum tuberosum L.). Plant Soil Environ. 53, 8, 355-360

Lachman, J., Hamouz, K., Orsak, M., Pivec, V., \& Dvorak, P. (2008). The influence of flesh colour and growing locality on polyphenolic content and antioxidant activity in potatoes. Sci Hortic. 117, 109-114

Leo, L., Leone, A., Longo, C., Lombardi, D. A., Raimo, F., \& Zacheo, G. (2008). Antioxidant compounds and antioxidant activity in "early potatoes". J Agric Food Chem. 56, 11, 4154-4163

Levine, M., Conry-Cantilena, C., Wang, Y., Welch, R. W., Washko, P. W., Dhariwal, K. R., Park, J. B., lazarev, A., Graumlich, J. F., King, J., \& Cantilena, L. R. (1996). Vitamin C pharmacokinetics in healthy volunteers: Evidence for a recommended dietary allowance. Proc Natl Acad Sci USA 93, 3704-3709 
Love, S. L. \& Salaiz, T. (2004). Stability of expression and concentration of ascorbic acid in North American potato germplasm. Hortscience. 39, 1, 156-160

Lu, W. \& Haynes, K. (2001). Carotenoid content and color in diploid potatoes. J Amer Soc Hort Sci. 126, 6, 722-726

Lukaszewicz, M., Matysiak-Kata, I., Skala, J., Fecka, I., Cisowski, W., \& Szopa, J. (2004). Antioxidant capacity manipulation in transgenic potato tuber by changes in phenolic compounds content. J Agric Food Chem. 52, 1526-1533

Mäder, J., Rawel, H., \& Kroh, L. W. (2009). Composition of phenolic compounds and glycoalkaloids $\alpha$-solanine and $\alpha$-chaconine during commercial potato processing. $J$ Agric Food Chem. 57, 14, 6292-6297

Madiwale, G. P., Reddivari, L., Holm, D. G., \& Vanamala, J. (2011). Storage elevates phenolic content and antioxidant activity but suppresses antiproliferative and pro-apoptotic properties of colored-flesh potatoes against human colon cancer cell lines. J Agric Food Chem. doi:10.1021/jf201073g,

Manach, C., Scalbert, A., Morand, C., Remesy, C., \& Jimenez, L. (2004). Polyphenols: food sources and bioavailability. Am J Clin Nutr. 79, 5, 727-747

Mattila, P. \& Hellström, J. (2007). Phenolic acids in potatoes, vegetables, and some of their products. J Food Compos Anal. 20, 152-160

Max-Rubner-Institut - Karlsruhe (2010), Bundeslebensmittelschlüssel, In: Bundesministerium für Ernährung, Landwirtschaft und Verbraucherschutz, 18-7-2011, Available from http://www.bls.nvs2.de/index.php?id=39

Morris, W. L., Ducreux, L., Griffiths, D. W., Stewart, D., Davies, H. V., \& Taylor, M. A. $(2004)$. Carotenogenesis during tuber development and storage in potato. J Exp Bot. $55,975-982$

Mulinacci, N., Ieri, F., Giaccherini, C., Innocenti, M., Andrenelli, L., Canova, G., Saracchi, M., \& Casiraghi, M. C. (2008). Effect of cooking on the anthocyanins, phenolic acids, glycoalkaloids, and resistant starch content in two pigmented cultivars of Solanum tuberosum L. J Agric Food Chem. 56, 24, 11830-11837

Natella, F., Belelli, F., Ramberti, A., \& Scaccini, C. (2010). Microwave and traditional cooking methods: Effect of cooking on antioxidant capacity and phenolic compounds content of seven vegetables. J Food Biochem. 34, 796-810

National Food Institute - Technical University of Denmark (16-3-2009), Nutrition Information and facts, In: Danish Food Composition Databank - ed.7.01, 18-7-2011, Available from http://www.foodcomp.dk/v7/fcdb_default.asp

Navarre, D. A., Pillai, S. S., Shakya, R., \& Holden, M. J. (2011). HPLC profiling of phenolics in diverse potato genotypes. Food Chem. 127, 34-41

Navarre, D. A., Shakya, R., Holden, J., \& Kumar, S. (2010). The effect of different cooking methods on phenolics and vitamin $\mathrm{C}$ in developmentally young potato tubers. Am J Pot Res. 87, 350-359

Nesterenko, S. \& Sink, K. C. (2003). Carotenoid profiles of potato breeding lines and selected cultivars. Hortscience. 38, 6, 1173-1177

Nutrient Data Laboratory (12-2-2010), USDA National Nutrient Database for Standard Reference, In: United States Department of Agriculture, 18-6-2011, Available from http:/ / www.nal.usda.gov/fnic/foodcomp/search/ 
Penarrieta, J. M., Salluca, T., Tejeda, L., Alvarado, J. A., \& Bergenstahl, B. (2011). Changes in phenolic antioxidants during chuno production (traditional Andean freeze and sun-dried potato). J Food Compos Anal. 24, 580-587

Pinhero, R. G., Coffin, R., and Yada, R. Y. (2009). Post-harvest storage of potatoes, In: Advances in Potato Chemistry and Technology, Singh, J. and Kaur, L., pp.(339-370), Elsevier Inc., 978-0-12-374349-7, Amsterdam

Qin, A., Shi, Q., \& Yu, X. (2011). Ascorbic acid contents in transgenic potato plants overexpressing two dehydroascorbate reductase genes. Mol Biol Rep. 38, 1557-1566

Reddivari, L., Hale, A. L., \& Miller, J. C., Jr. (2007). Genotype, location, and year influence antioxidant activity, carotenoid content, phenolic content, and composition in specialty potatoes. J Agric Food Chem. 55, 8073-8079

Rembialkowska, E. (1998). [A comparison of selected parameters of potatoes health quality from ecologically oriented and conventional farms]. Rocz Panstw Zakl Hig. 49, 2, 159-167

Reyes, L. F., Miller, J. C., Jr., \& Cisneros-Zevallos, L. (2004). Environmental conditions influence the content and yield of anthocyanins and total phenolics in pruple- and red-flesh potatoes during tuber development. Am J Potato Res. 81, 187-193

Robert, L., Narcy, A., Rayssiguier, Y., Mazur, A., \& Remesy, C. (2008). Lipid metabolism and antioxidant status in sucrose vs. potato-fed rats. J Am Coll Nutr. 27, 1, 109-116

Robert, L., Narcy, A., Rock, E., Demigne, C., Mazur, A., \& Remesy, C. (2006). Entire potato consumption improves lipid metabolism and antioxidant status in cholesterol-fed rat. Eur J Nutr. 45, 5, 267-274

Rodriguez, G. B., Rios, M. D., Rodriguez Rodriguez, E. M., \& Diaz, R. C. (2010). Influence of the cultivar on the organic acid and sugar composition of potatoes. J Sci Food Agric. 90, 13, 2301-2309

Rogan, G. J., Bookout, J. T., Duncan, D. R., Fuchs, R. L., Lavrik, P. B., Love, S. L., Mueth, M., Olson, T., Owens, E. D., Raymond, P. J., \& Zalewski, J. (2000). Compositional analysis of tubers from insect and virus resistant potato plants. J Agric Food Chem. 48, 5936-5945

Rommens, C. M., Richael, C. M., Yan, H., Navarre, D. A., Ye, J., Krucker, M., \& Swords, K. (2008). Engineered native pathways for high kaempferol and caffeoylquinate production in potato. Plant Biotechnol J. 6, 9, 870-886

Rumbaoa, R. G. O., Cornago, D. F., \& Geronimo, I. M. (2009). Phenolic content and antioxidant capacity of Philippine potato (Solanum tuberosum) tubers. J Food Compos Anal. 22, 546-550

Scalbert, A. \& Williamson, G. (2000). Dietary intake and bioavailability of polyphenols. J Nutr. Supplement, 2073S-2085S

Shakya, R. \& Navarre, D. A. (2006). Rapid screening of ascorbic acid, glycoalkaloids, and phenolics in potato using high-performance liquid chromatography. J Agric Food Chem. 54, 5253-5260

Singh, A., Sabally, K., Kubow, S., Donnely, D. J., Gariepy, Y., Orsat, V., \& Raghavan, G. S. V. (2011). Microwave-assisted extraction of phenolic antioxidants from potato peels. Molecules. 16, 2218-2232

Singh, J. and Kaur, L. (2009). Advances in potato chemistry and technology, In: Elsevier, Burlington, USA 
Soltoft, M., Nielsen, J., Holst, L. K., Husted, S., Halekoh, U., \& Knuthsen, P. (2010). Effects of organic and conventional growth systems on the content of flavonoids in onions and phenolic acids in carrots and potatoes. J Agric Food Chem. 58, 19, 10323-10329

Spooner, D. M., Nunez, J., Trujillo, G., Herrera, M. R., Guzman, F., \& Ghislain, M. (2007). Extensive simple sequence repeat genotyping of potato landraces supports a major reevaluation of their gene pool structure and classification. Proc Natl Acad Sci USA $104,49,19398-19403$

Spychalla, J. P. \& Desborough, S. L. (1990). Superoxide dismutase, catalase, and alphatocopherol content of store potato tubers. Plant Physiol. 94, 1214-1218

Stalmach, A., Steiling, H., Williamson, G., \& Crozier, A. (2010). Bioavailability of chlorogenic acids following acute ingestion of coffee by humans with an ileostomy. Arch Biochem Biophys. 501, 1, 98-105

Stevenson, D. E. \& Hurst, R. D. (2007). Polyphenolic phytochemicals-just antioxidants or much more? Cell Mol Life Sci. 64, 2900-2916

Stratil, P., Klejdus, B., \& Kuban, V. (2006). Determination of total content of phenolic compounds and their antioxidant activity in vegetables--evaluation of spectrophotometric methods. J Agric Food Chem. 54, 3, 607-616

Stushnoff, C., Ducreux, L. J. M., Hancock, R. D., Hedley, P. E., Holm, D. G., McDougall, G. J., McNicol, J. W., Morris, J., Morris, W. L., Sungurtas, J. A., Verrall, S. R., Zuber, T., \& Taylor, M. A. (2010). Flavonoid profiling and transcriptome analysis reveals new gene-metabolite correlations in tubers of Solanum tuberosum L. J Exp Bot. 61, 4, 12251238

Thompson, M. D., Thompson, H. J., mcGinley, J. N., Neil, E. S., Rush, D. K., Holm, D. G., \& Stushnoff, C. (2009). Functional food characteristics of potato cultivars (Solanum tuberosum L.): phytochemical composition and inhibition of 1-methyl-1nitrosurea induced breast cancer in rats. J Food Compos Anal. 22, 571-576

Tudela, J. A., Cantos, E., Espín, J. C., Tomás-Barberán, F. A., \& Gil, M. A. (2002). Induction of antioxidant flavonol biosynthesis in fresh-cut potatoes. Effect of domestic cooking. $J$ Agric Food Chem. 50, 5925-5931

White, P. J. \& Broadley, M. R. (2005). Biofortifying crops with essential mineral elements. Trends Plant Sci. 10, 12, 586-593

Williamson, G. \& Manach, C. (2005). Bioavailability and bioefficacy of polyphenols in humans. II. review of 93 intervention studies. Am J Clin Nutr. 81, 243S-255S

Woolfe, J. A. (1987). Effects of storage, cooking and processing on the nutritive value of potatoes, In: Woolfe, J. A., pp. (83-161), Cambridge University Press, Cambridge

Xu, X., Li, W., Lu, Z., Beta, T., \& Hydamaka, A. W. (2009). Phenolic content, composition, antioxidant activity, and their changes during domestic cooking of potatoes. J Agric Food Chem. 57, 21, 10231-10238

Yun, S., Habicht, J.-P., Miller, D. D., \& Glahn, R. P. (2004). An in vitro digestion/Caco-2 cell culture system accurately predicts the effects of ascorbic acid and polyphenolic compounds on iron bioavailability in humans. J Nutr. 134, 2717-2721

Zhou, X., McQuinn, R., Fei, Z., Wolters, A.-M. A., van Eck, J., Brown, C., Giovannoni, J. J., \& Li, L. (2011). Regulatory control of high levels of carotenoid accumulation in potato tubers. Plant Cell Environ. 34, 1020-1030 
Zhu, F., Cai, Y. Z., Ke, J., \& Corke, H. (2010). Compositions of phenolic compounds, amino acids and reducing sugars in commercial potato varieties and their effects on acrylamide formation. J Sci Food Agric. 90, 13, 2254-2262 


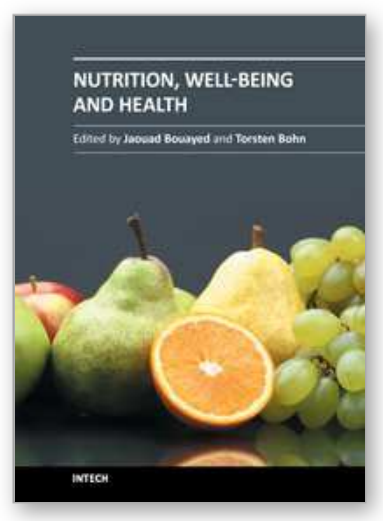

\author{
Nutrition, Well-Being and Health \\ Edited by Dr. Jaouad Bouayed
}

ISBN 978-953-51-0125-3

Hard cover, 224 pages

Publisher InTech

Published online 23, February, 2012

Published in print edition February, 2012

In our modern society, expectations are high, also with respect to our daily diet. In addition to being merely "nutritious", i.e. supplying a variety of essential nutrients, including macro-nutrients such as proteins or micronutrients such as minerals and vitamins, it is almost expected that a good diet offers further advantages especially well-being and health and the prevention of chronic diseases, which are, as we generally tend to grow older and older, becoming a burden to enjoying private life and to the entire society. These additional qualities are often sought in diets rich also in non-nutritive components, such as phytochemicals. In contrast to drugs, which are taken especially to cure or ameliorate diseases, it is expected that a healthy diet acts in particular on the side of prevention, allowing us to become old without feeling old. In the present book, rather then trying to give an exhaustive overview on nutritional aspects and their link to well-being and health, selected topics have been chosen, intended to address presently discussed key issues of nutrition for health, presenting a reasonable selection of the manifold topics around diet, well-being, and health: from the antioxidants polyphenols and carotenoids, aroma-active terpenoids, to calcium for bone health, back to traditional Chinese Medicine.

\title{
How to reference
}

In order to correctly reference this scholarly work, feel free to copy and paste the following:

Danièle Evers and Hannah Deußer (2012). Potato Antioxidant Compounds: Impact of Cultivation Methods and Relevance for Diet and Health, Nutrition, Well-Being and Health, Dr. Jaouad Bouayed (Ed.), ISBN: 978-953-510125-3, InTech, Available from: http://www.intechopen.com/books/nutrition-well-being-and-health/potatoantioxidant-compounds-impact-of-cultivation-methods-and-relevance-for-diet-and-health

\section{INTECH}

open science | open minds

\section{InTech Europe}

University Campus STeP Ri

Slavka Krautzeka 83/A

51000 Rijeka, Croatia

Phone: +385 (51) 770447

Fax: +385 (51) 686166

www.intechopen.com

\section{InTech China}

Unit 405, Office Block, Hotel Equatorial Shanghai

No.65, Yan An Road (West), Shanghai, 200040, China

中国上海市延安西路65号上海国际贵都大饭店办公楼 405 单元

Phone: +86-21-62489820

Fax: +86-21-62489821 
(C) 2012 The Author(s). Licensee IntechOpen. This is an open access article distributed under the terms of the Creative Commons Attribution 3.0 License, which permits unrestricted use, distribution, and reproduction in any medium, provided the original work is properly cited. 